\title{
Supporting Information (SI): How Microscopic Features of Mineral Surfaces Critically Influence Heterogeneous Ice Nucleation
}

\author{
Abhishek Soni and G. N. Patey* \\ Department of Chemistry, University of British Columbia, Vancouver, British Columbia, \\ Canada V6T $1 Z 1$ \\ E-mail: patey@chem.ubc.ca
}




\section{Contents}

- Figures S1 - S3 and Table S1: Crystallographic information for the physical systems investigated.

- Figure S4: NPT simulation for Hematite showing the hexagonal water monolayer, and the size of the triangular lattice formed.

- Figures S5 - S7: Water oxygen atom density profiles perpendicular to various scaled surfaces.

- Figure S8: Water oxygen and hydrogen atom density profiles perpendicular to AgI and $\mathrm{PbI}_{2}$ surfaces.

- Figure S9: NPT simulation results at $230 \mathrm{~K}$ for the boehmite (010) surface.

- Table S2: The complete set of $\delta$ lattice mismatch values for all physical surfaces considered.

- Table S3: Summary of the NPT simulations at $230 \mathrm{~K}$ for all physical systems considered.

- Tables S5 - S6: Summaries of all other NPT and NVT simulations including scaled surfaces.

- Tables S7 - S8: Summary of surface measurements used to calculate $\delta_{2 D}$. Values of $\delta$ and $\delta_{2 D}$ for the basal and prism planes of ice, summarized for all surfaces considered.

- Table S9: Distances related to the size of the interstitial sites that influence the heterogeneous ice nucleation capabilities of kaolinite and gibbsite. 


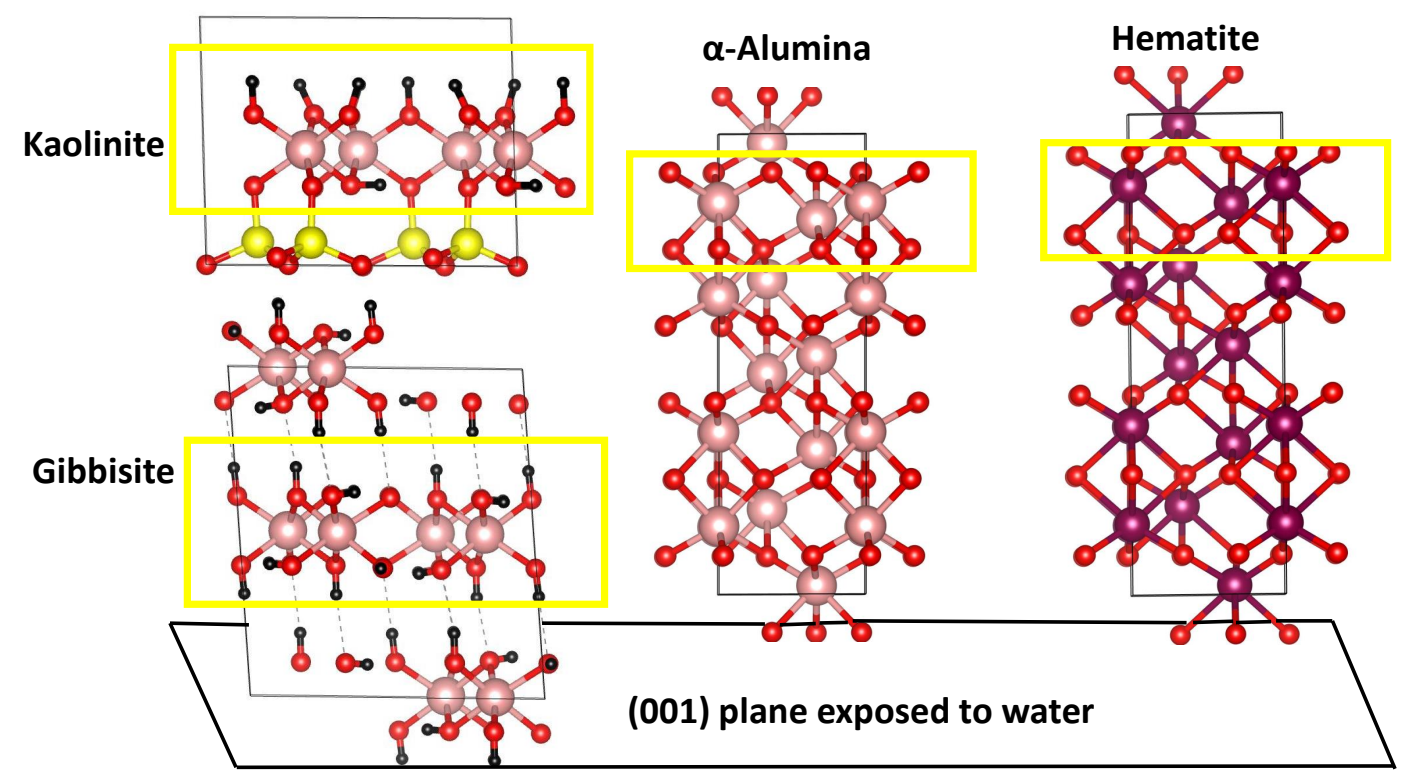

Figure S1: Unit cell representations of kaolinite, gibbsite, $\alpha$-alumina, and hematite. The regions outlined in yellow are the basic units from which larger (001) slabs are created by periodic repetition in the $\mathrm{x}$ and $\mathrm{y}$ directions. Note that the Si surface is removed in the kaolnoSi slab. For gibbsite some simulations were performed using slabs twice as thick as the yellow outline, but no significant dependence on thickness was observed. All surface oxygen atoms are protonated (not shown). The Al, Fe, Si, O, and H atoms are colored, pink, grey, yellow, red, and black, respectively. 


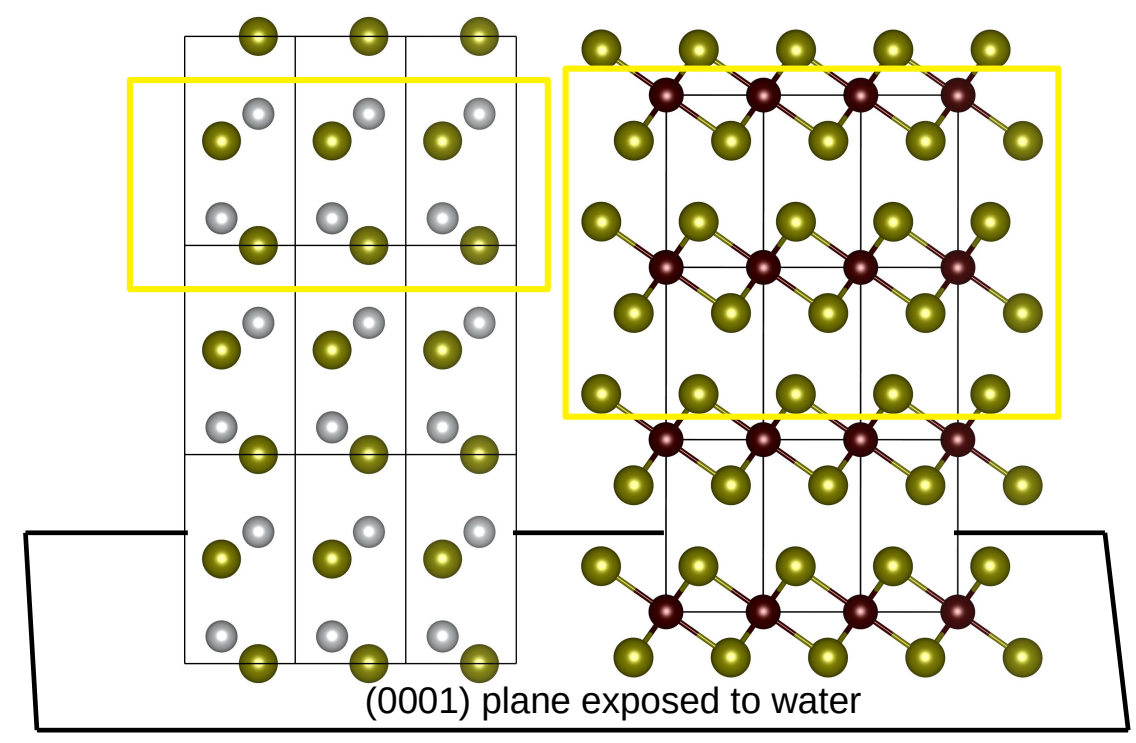

Figure S2: Unit cell representations of $\beta-\mathrm{AgI}$ and $\mathrm{PbI}_{2}$. The regions outlined in yellow are the basic units from which larger (0001) slabs are created by periodic repetition in the $\mathrm{x}$ and y directions. The $\mathrm{Ag}, \mathrm{Pb}$, and I atoms are colored silver, black, and green, respectively. 


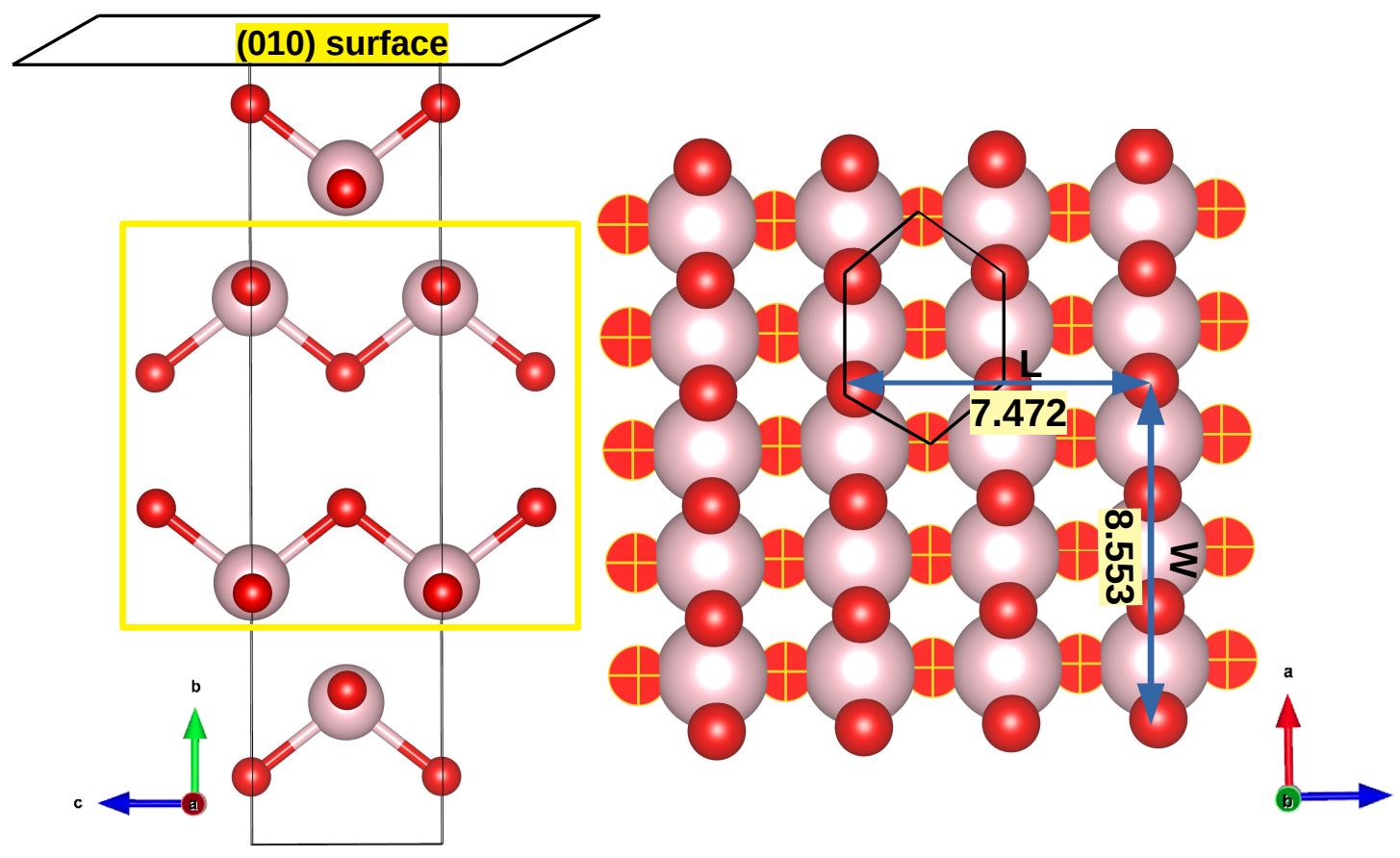

Figure S3: The left panel is a side view of the unit cell of boehmite $(\gamma-\mathrm{AlO}(\mathrm{OH}))$. The right panel is a top view of the (010) plane of boehmite. The region outlined in yellow is the basic unit from which larger (010) slabs are created by periodic repetition in the $\mathrm{x}$ and y directions. The hexagons outlined in black have four $\mathrm{O}$ atoms in the top layer, and two in the bottom (denoted with + ). All $\mathrm{O}$ atoms in the top layer are protonated (not shown). Al and $\mathrm{O}$ atoms are colored pink and red, respectively. Measurements are given in $\AA$. 
Table S1: Unit cell parameters for experimental crystal structures.

\begin{tabular}{cccccccc} 
Crystal & Unit cell & $\mathrm{a}(\AA)$ & $\mathrm{b}(\AA)$ & $\mathrm{c}(\AA)$ & $\alpha$ & $\beta$ & $\gamma$ \\
\hline Ice $^{1,2}$ & Hexagonal & 4.506 & 4.506 & 7.346 & 90 & 90 & 120 \\
$\alpha$-alumina & \\
Hematite $^{4}$ & Hexagonal & 4.75 & 4.75 & 12.98 & 90 & 90 & 120 \\
Kaolinite $^{5}$ & Triclinic & 5.15 & 8.94 & 7.39 & 91.93 & 105.05 & 89.80 \\
Gibbsite $^{6}$ & Monoclinic & 8.69 & 5.08 & 9.74 & 90 & 90.54 & 90 \\
$\beta$-AgI $^{7}$ & Hexagonal & 4.60 & 4.60 & 7.51 & 90 & 90 & 120 \\
$\mathrm{PbI}_{2}{ }^{8}$ & Hexagonal & 4.56 & 4.56 & 20.94 & 90 & 90 & 120 \\
Boehmite $^{9}$ & Orthorhombic & 2.85 & 12.12 & 3.74 & 90 & 90 & 90
\end{tabular}




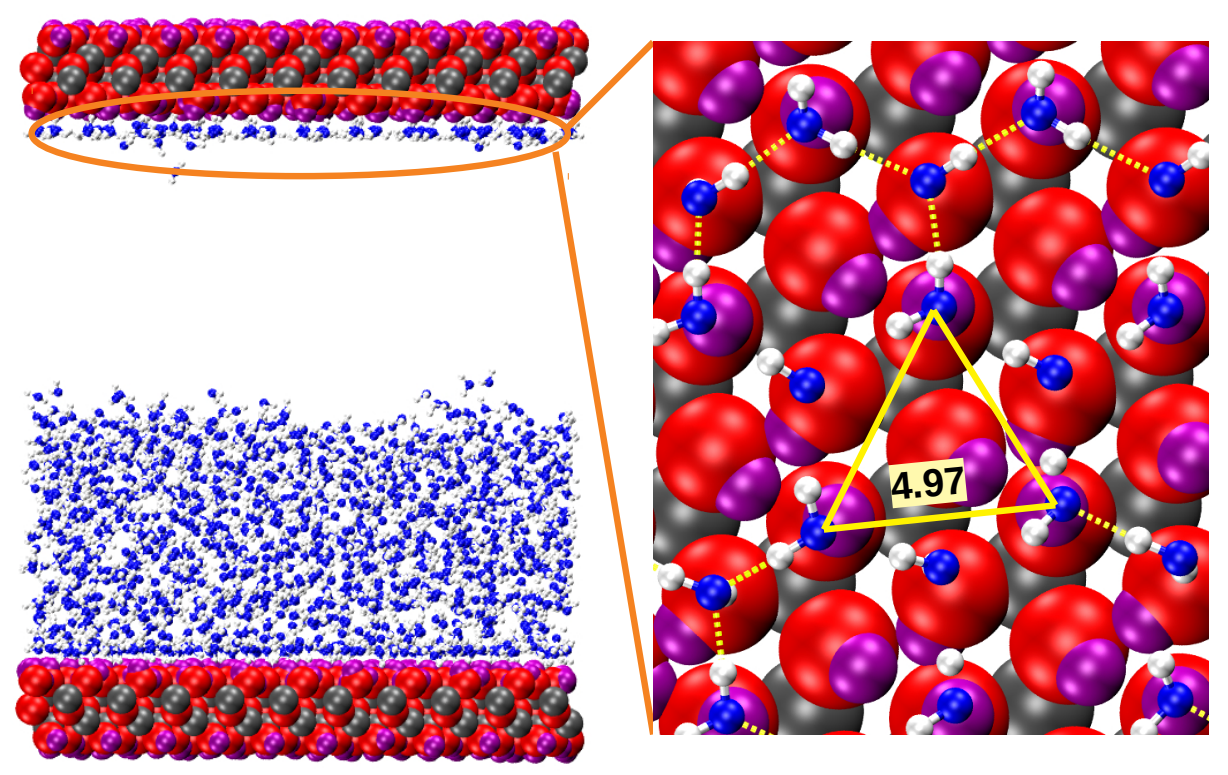

Figure S4: The left panel indicates that the water near the hematite surface under NPT conditions at $230 \mathrm{~K}$ shows no hint of ice structure. The right panel shows the water monolayer that has condensed onto the upper hematite surface. Note that in the monolayer the measured triangular lattice distance between the water $\mathrm{O}$ atoms $(\sim 4.97 \AA)$ is larger than that of ice $(4.506 \AA)$. The water monolayer does exhibit hexagonal structure, but not the systematic water-water hydrogen bonding characteristic of smaller ice hexagons. Water $\mathrm{O}$ and $\mathrm{H}$ atoms are colored blue and white, respectively. The Fe, $\mathrm{O}$, and $\mathrm{H}$ atoms of the hematite slab are colored grey, red, and purple, respectively. Hydrogen bonds are shown as dotted yellow lines. 

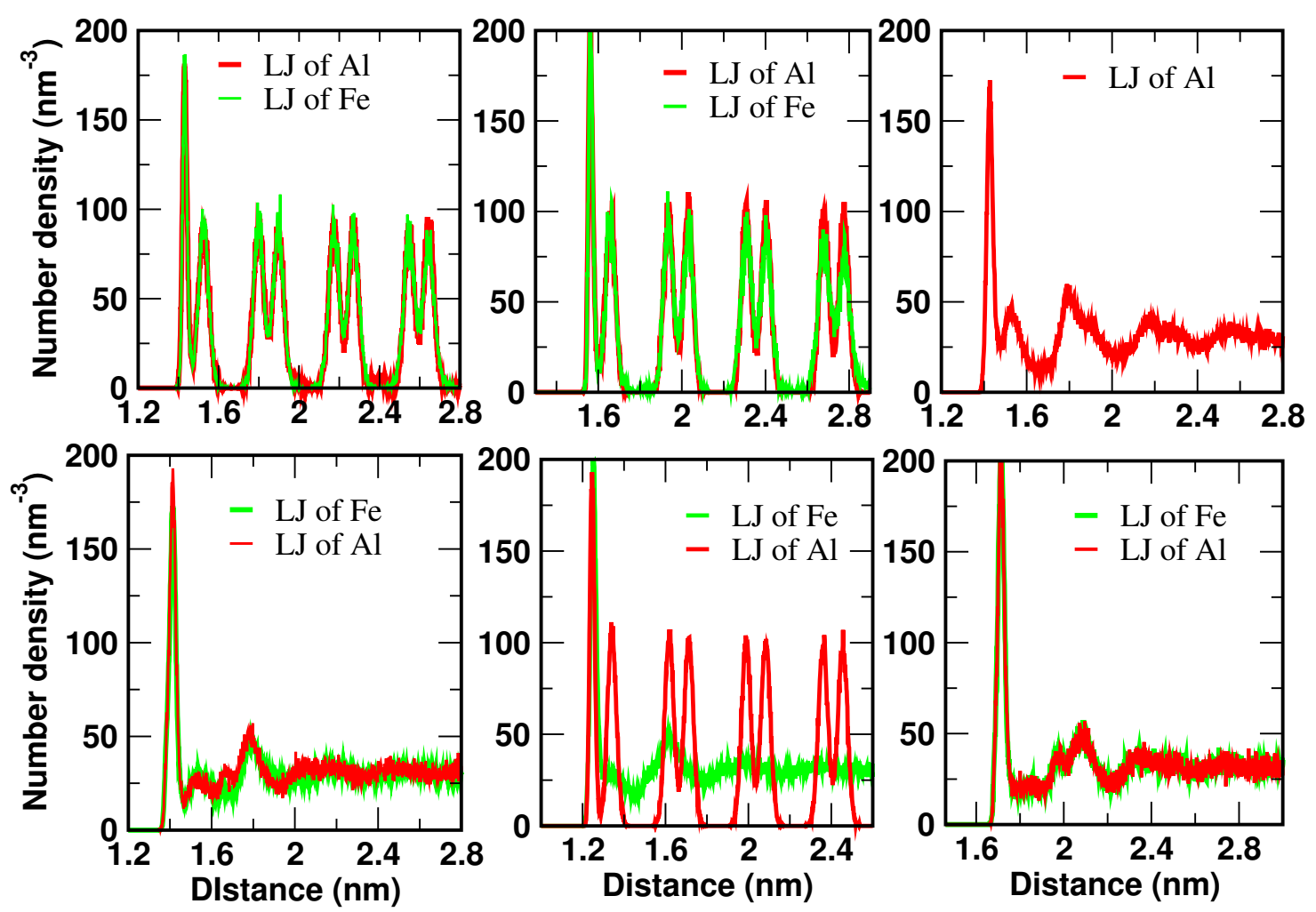

Figure S5: Water O atom density profiles. Top panel: $\alpha$-alumina (left), $\alpha$-alumina scaled to ice (AsI) (middle), and $\alpha$-alumina scaled to hematite (AsH) (right). Bottom panel: hematite (left), hematite scaled to ice (HsI) (middle), and hematitle scaled to alumina (HsA) (right). The red and green curves were obtained with the $\mathrm{Al}$ and $\mathrm{Fe}$ force fields, respectively. 

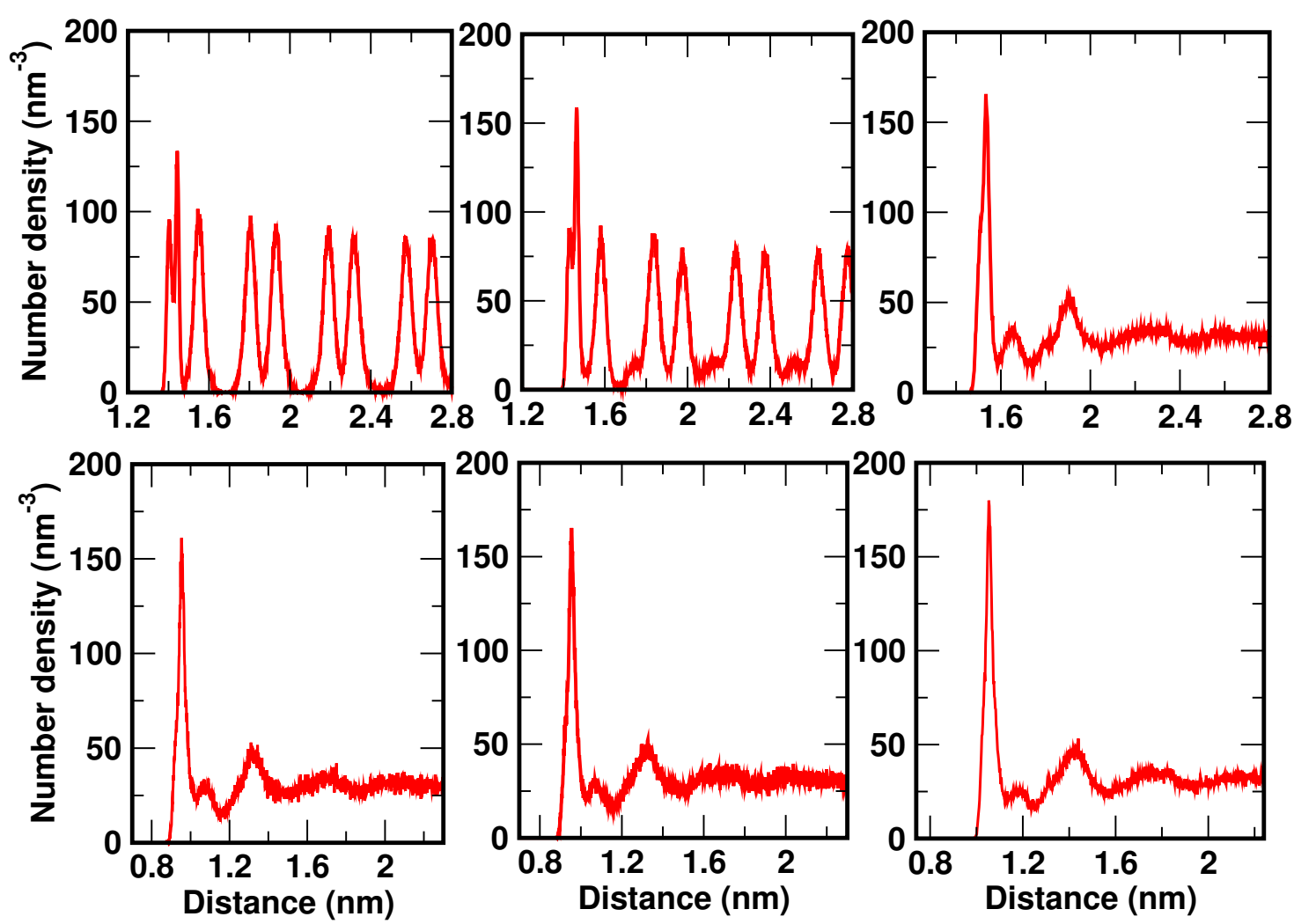

Figure S6: Water O atom density profiles. Top panel: kaolnoSi (left), kaolnoSi scaled to gibbsite (KsG) (middle,) and kaolnoSi scaled to ice (KsI) (right). Bottom panel: gibbsite (left), gibbsite scaled to kaolnoSi (GsK) (middle), and gibbsite scaled to ice (GsI) (right). 

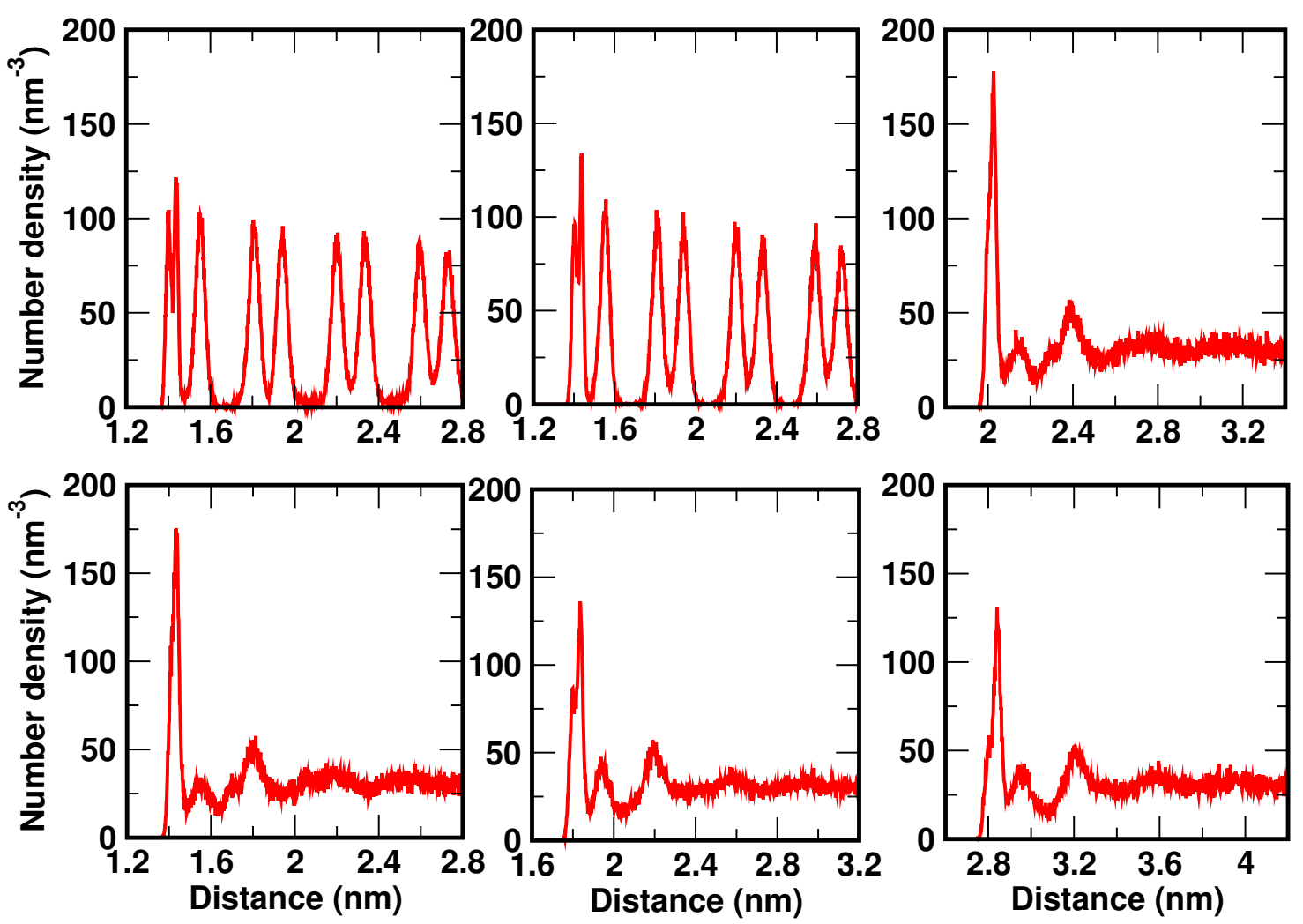

Figure S7: Water O atom density profiles. Top panel: Kc1 (left), Kc2 (middle), and Kc3 (right). Bottom panel: Kc4 (left), Ke (middle), and Ge (right). 


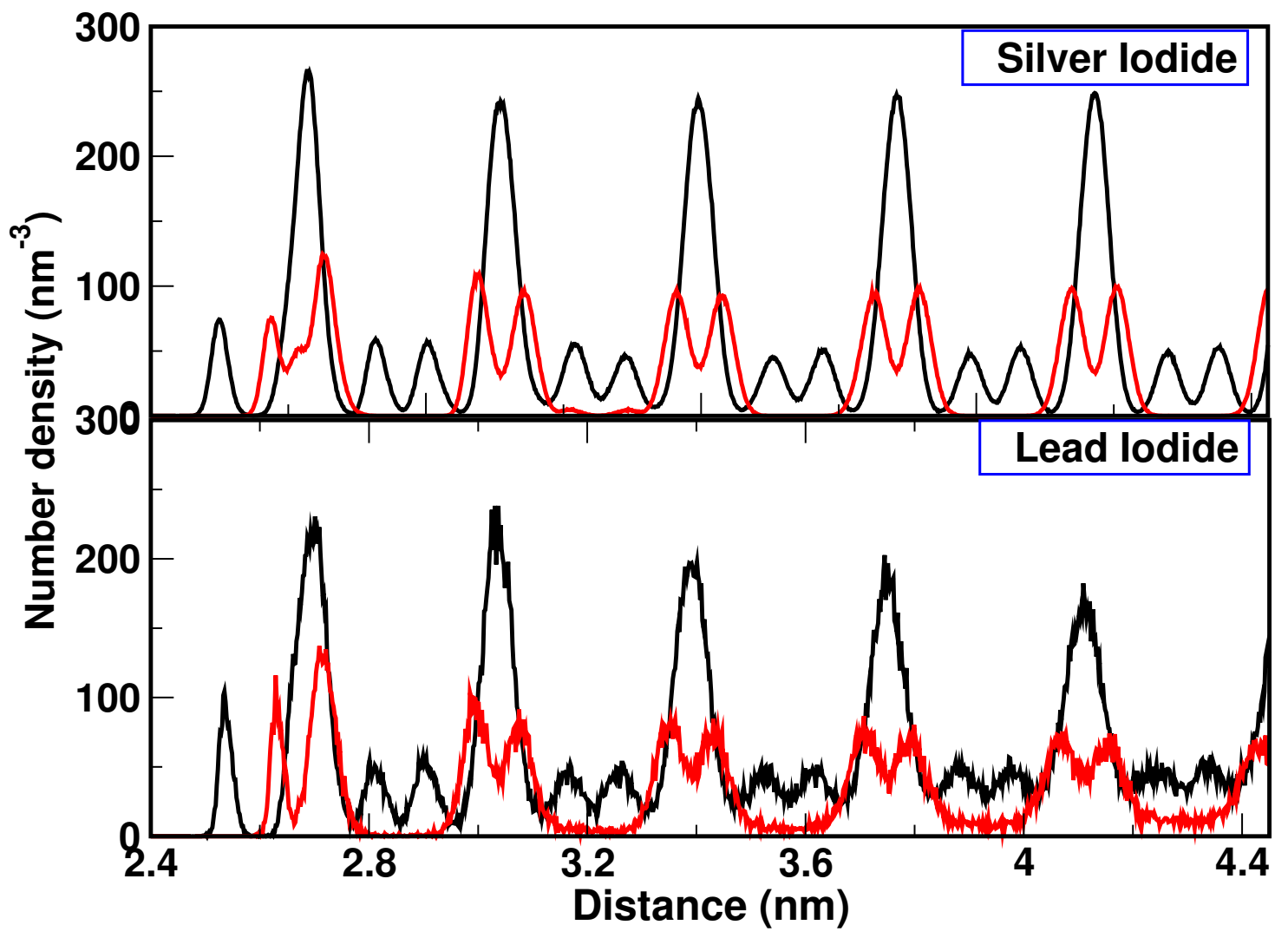

Figure S8: Density profiles of water $\mathrm{O}$ (red) and $\mathrm{H}$ (black) atoms perpendicular to the (0001) surfaces of $\beta$ - $\mathrm{AgI}$ and $\mathrm{PbI}_{2}$. 


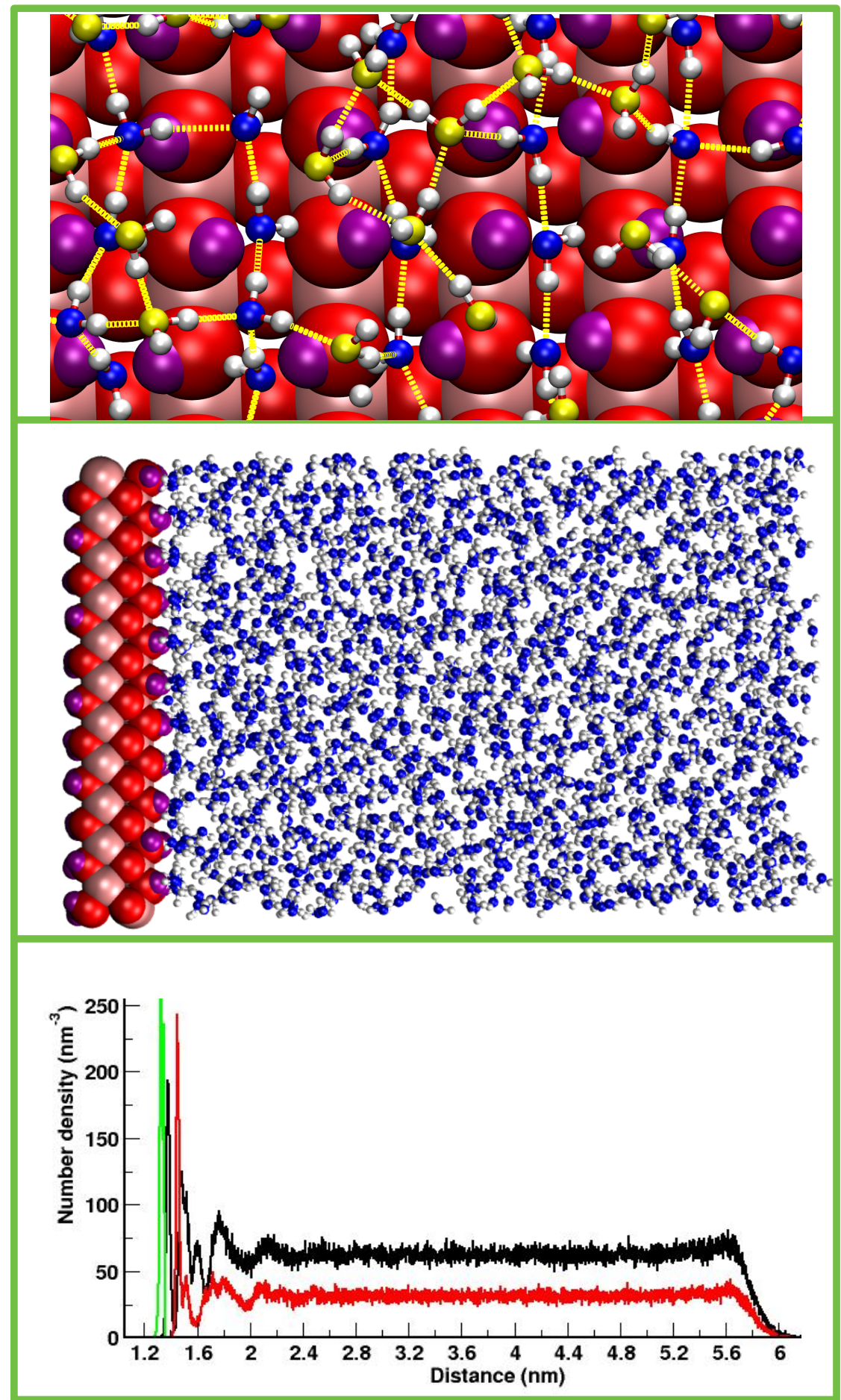

Figure S9: Top panel: Water structure on the boehmite $(\gamma$ - $\mathrm{AlO}(\mathrm{OH}))(010)$ surface at 230 K. Middle panel: Side view of water at the boehmite surface. Bottom panel: Water O (red) and H (black) atom density profiles perpendicular to the boehmite (010) surface. The density profile of the surface hydroxyl $\mathrm{H}$ atoms is shown as a green curve. Atoms are colored as in Figure S3 and S4. 
Table S2: $\delta$ mismatch parameters for the surfaces considered. The lattice parameters are given in parentheses, and the values of $\mathrm{n}$ and $\mathrm{m}$ are in square brackets. Note that for $\mathrm{I}_{h}$, the $\mathrm{a}$ and $\mathrm{b}$ unit cell parameters are equivalent. For $\mathrm{I}_{c}$ all three axes are equivalent, and the lattice parameter for the (111) plane is used.

\begin{tabular}{|c|c|c|}
\hline Lattice parameter $\alpha-A$ & Alumina-a,b (4.754 $\AA$ ) & Hematite-a,b $(5.03 \AA)$ \\
\hline $\mathrm{I}_{h}-\mathrm{a}(4.506 \AA)$ & {$[1: 1] 5.5 \%$} & [1:1] $11.6 \%$ \\
\hline $\mathrm{I}_{h^{-}} \mathrm{c}(7.346 \AA)$ & {$[2: 3] 2.93 \%$} & {$[2: 3] 2.71 \%$} \\
\hline $\mathrm{I}_{c}(4.504 \AA)$ & [1:1] $5.55 \%$ & {$[1: 1] 11.68 \%$} \\
\hline Lattice parameter & Kaolinite-a $(5.15 \AA)$ & Kaolinite-b (8.94 A) \\
\hline $\mathrm{I}_{h^{-}} \mathrm{a}(4.506 \AA)$ & {$[1: 1] 14.3 \%$} & {$[2: 1] 0.8 \%$} \\
\hline $\mathrm{I}_{h^{-}} \mathrm{c}(7.346 \AA)$ & [2:3] $5.16 \%$ & [1:1] $21.7 \%$ \\
\hline $\mathrm{I}_{c}(4.504 \AA)$ & {$[1: 1] 14.34 \%$} & {$[2: 1] 0.75 \%$} \\
\hline Lattice parameter & Gibbsite-a $(8.684 \AA)$ & Gibbsite-b $(5.078 \AA)$ \\
\hline $\mathrm{I}_{h^{-}} \mathrm{a}(4.506 \AA)$ & [2:1] $3.6 \%$ & {$[1: 1] 12.7 \%$} \\
\hline $\mathrm{I}_{h^{-}} \mathrm{c}(7.346 \AA)$ & {$[1: 1] 18.21 \%$} & [2:3] $3.69 \%$ \\
\hline $\mathrm{I}_{c}(4.504 \AA)$ & [2:1] $3.6 \%$ & {$[1: 1] 12.74 \%$} \\
\hline Lattice parameter & r AgI-a,b (4.598 A) & $\mathrm{PbI}_{2}-\mathrm{a}, \mathrm{b}(4.557 \AA)$ \\
\hline $\mathrm{I}_{h^{-}} \mathrm{a}(4.506 \AA)$ & {$[1: 1] 2.04 \%$} & {$[1: 1] 1.13 \%$} \\
\hline $\mathrm{I}_{h^{-}} \mathrm{c}(7.346 \AA)$ & [2:3] $6.11 \%$ & {$[2: 3] 6.95 \%$} \\
\hline $\mathrm{I}_{c}(4.504 \AA)$ & [1:1] $2.09 \%$ & [1:1] $1.18 \%$ \\
\hline Lattice parameter & Boehmite-a (2.851 A) & Boehmite-c (3.736 A) \\
\hline $\mathrm{I}_{h}-\mathrm{a}(4.506 \AA)$ & {$[1: 2] 26.5 \%$} & {$[1: 1] 17 \%$} \\
\hline $\mathrm{I}_{h}-\mathrm{c}(7.346 \AA)$ & {$[1: 3] 16.43 \%$} & {$[1: 2] 1.72 \%$} \\
\hline $\mathrm{I}_{c}(4.504 \AA)$ & {$[1: 2] 26.6 \%$} & {$[1: 1] 17.05 \%$} \\
\hline
\end{tabular}


Table S3: Summary of NPT simulations performed with the TIP4P/Ice water model at 230 $\mathrm{K} . \mathrm{x}, \mathrm{y}$, and $\mathrm{z}$ are the dimensions of the simulation cell, $\mathrm{N}$ is the number of water molecules in the central cell, Trials are the number of simulations run with different initial conditions, and Time is the length of the simulation. The numbers in parentheses in the Trials column are the number of of trials where ice nucleation was observed. The numbers in parentheses in the Time column are the starting times for ice nucleation. Note that $\mathrm{z}$ includes two vacuum spaces, each $1.0 \mathrm{~nm}$ thick, as measured from the boundary of the slab unit cell.

\begin{tabular}{cccccccc} 
Surface Label & $\mathrm{N}$ & Trials & Time $(\mathrm{ns})$ & Ice Nucleated $(\mathrm{IN})$ & $\mathrm{x}(\mathrm{nm})$ & $\mathrm{y}(\mathrm{nm})$ & $\mathrm{z}(\mathrm{nm})$ \\
\hline$\alpha$-alumina & 1320 & $4(4)$ & $1100(600)$ & Yes & 3.8 & 3.3 & 7 \\
Hematite & 1600 & $3(0)$ & 6300 & No & 5 & 4.33 & 7 \\
Kaolinite & 1450 & $2(2)$ & $800(400)$ & Yes & 3.6 & 3.57 & 10 \\
KaolnoSi & 1450 & $3(3)$ & $1100(430)$ & Yes & 3.6 & 3.57 & 10 \\
Gibbsite & 1000 & $4(0)$ & 10000 & No & 3.5 & 3.05 & 7 \\
$\beta$-AgI (Ag exposed) & 1350 & $2(2)$ & $200(80)$ & Yes & 3.2 & 2.78 & 10 \\
$\beta$-AgI (I exposed) & 1350 & $2(0)$ & 3000 & No & 3.2 & 2.78 & 10 \\
$\mathrm{PbI}_{2}$ (Pb exposed) & 1350 & $2(2)$ & $200(60)$ & Yes & 3.2 & 2.78 & 10 \\
$\mathrm{PbI}_{2}$ (I exposed) & 1350 & $2(0)$ & 3000 & No & 3.2 & 2.78 & 10 \\
Boehmite & 1670 & $3(0)$ & 9500 & No & 3.15 & 9.5 & 3.73
\end{tabular}


Table S4: Summary of other simulations performed for $\alpha$-alumina, hematite, and scaled surfaces. (LJ Al) and (LJ Fe) indicate the force field used. In the CLAYFF model Al and Fe carry the same partial charges, such that only their LJ parameters differ. Everything else is as in Table S5.

\begin{tabular}{|c|c|c|c|c|c|c|c|c|}
\hline Surface Label & $\mathrm{N}$ & $\rho(\mathrm{g} / \mathrm{cc})$ & Trials & Time(ns) & $\mathrm{IN}$ & $\mathrm{x}(\mathrm{nm})$ & $\mathrm{y}(\mathrm{nm})$ & $\mathrm{z}(\mathrm{nm})$ \\
\hline$\alpha$-Alumina & $1690(\mathrm{NVT})$ & 0.945 & $2(2)$ & $2000(520)$ & Yes & 3.8 & 3.3 & 7 \\
\hline Alumina (LJ Fe) & $1690(\mathrm{NVT})$ & 0.945 & $3(3)$ & $2000(500)$ & Yes & 3.8 & 3.3 & 7 \\
\hline Alumina (LJ Fe) & $1320(\mathrm{NPT})$ & - & $2(2)$ & $2000(450)$ & Yes & 3.8 & 3.3 & 7 \\
\hline AsI & $1280(\mathrm{NPT})$ & - & $2(2)$ & $2000(360)$ & Yes & 3.15 & 2.13 & 8 \\
\hline AsI (LJ Fe) & 1280 (NPT) & - & $2(2)$ & $2000(310)$ & Yes & 3.15 & 2.13 & 8 \\
\hline $\mathrm{AsH}$ & 1445 (NVT) & - & $3(0)$ & 10000 & No & 3.5 & 3.03 & 7 \\
\hline $\mathrm{AsH}$ & $1250(\mathrm{NPT})$ & - & $2(0)$ & 9000 & No & 3.5 & 3.03 & 9 \\
\hline Hematite & $2926(\mathrm{NVT})$ & 0.925 & $3(0)$ & 5600 & No & 5 & 4.33 & 7 \\
\hline Hematite & $1600(\mathrm{NPT})$ & - & $2(0)$ & 6300 & No & 5 & 4.33 & 7 \\
\hline Hematite (LJ Al) & $3065(\mathrm{NVT})$ & 0.950 & $1(0)$ & 4350 & No & 5 & 4.33 & 7 \\
\hline Hematite (LJ Al) & $1700(\mathrm{NPT})$ & - & $2(0)$ & 4800 & No & 5 & 4.33 & 7 \\
\hline HsI & $1130(\mathrm{NPT})$ & - & $5(0)$ & 5600 & No & 3.16 & 2.74 & 8 \\
\hline HsI (LJ Al) & $1150(\mathrm{NPT})$ & - & $2(1)$ & $4500(500)$ & Yes & 3.16 & 2.74 & 8 \\
\hline Hc1 & 1160 (NPT) & - & $2(2)$ & $1780(600)$ & Yes & 3.1 & 2.68 & 8 \\
\hline Hc1 (LJ Al) & $1160(\mathrm{NPT})$ & - & $2(2)$ & $1800(250)$ & Yes & 3.1 & 2.68 & 8 \\
\hline Hc2 & 1120 (NPT) & - & $3(1)$ & $12000(4200)$ & Yes & 3.02 & 2.62 & 8 \\
\hline Hc2 (LJ Al) & 1120 (NPT) & - & $2(1)$ & $12000(1900)$ & Yes & 3.02 & 2.62 & 8 \\
\hline HsA & $2765(\mathrm{NVT})$ & 0.957 & $3(0)$ & 5600 & No & 4.75 & 4.11 & 7.5 \\
\hline HsA (LJ Al) & 2765 (NVT) & 0.96 & $4(0)$ & 8800 & No & 4.75 & 4.11 & 7.5 \\
\hline HsA (LJ Al) & $1660(\mathrm{NPT})$ & - & $2(0)$ & 8400 & No & 4.75 & 4.11 & 7.5 \\
\hline
\end{tabular}


Table S5: Summary of other simulations performed for kaolinite, kaolnoSi, gibbsite, and scaled surfaces. Both NPT and NVT simulations are listed. For NVT simulations $\rho$ is the bulk density of liquid water at $300 \mathrm{~K}$. Everything else is as in Table S3. In parenthesis, "small" and "big" indicate different surface dimensions, and "thick" indicates a gibbsite slab twice as thick as that shown in Figure S1.

\begin{tabular}{ccccccccc} 
Surface Label & $\mathrm{N}$ & $\rho(\mathrm{g} / \mathrm{cc})$ & Trials & Time $(\mathrm{ns})$ & $\mathrm{IN}$ & $\mathrm{x}(\mathrm{nm})$ & $\mathrm{y}(\mathrm{nm})$ & $\mathrm{z}(\mathrm{nm})$ \\
\hline Kaolinite & $1780(\mathrm{NVT})$ & 0.95 & $2(2)$ & $800(400)$ & Yes & 3.6 & 3.57 & 7 \\
KaolnoSi & $1780(\mathrm{NVT})$ & 0.95 & $3(3)$ & $1100(430)$ & Yes & 3.6 & 3.57 & 7 \\
KsI (small) & $1490(\mathrm{NPT})$ & - & $3(0)$ & 8000 & No & 3.4 & 3.6 & 10 \\
KsI (big) & $2100(\mathrm{NPT})$ & - & $2(0)$ & 19000 & No & 3.9 & 4.47 & 10 \\
KsG & $1692(\mathrm{NVT})$ & 0.945 & $5(2)$ & $10000(5015)$ & Yes & 3.55 & 3.45 & 7 \\
Kc1 & $1300(\mathrm{NPT})$ & - & $2(2)$ & $7000(2100)$ & Yes & 3.58 & 3.54 & 8 \\
Kc2 & $1300(\mathrm{NPT})$ & - & $2(2)$ & $7000(3200)$ & Yes & 3.54 & 3.50 & 8 \\
Kc3 & $1692(\mathrm{NPT})$ & - & $2(0)$ & 6000 & No & 3.48 & 3.45 & 8 \\
Kc4 & $1200(\mathrm{NPT})$ & - & $2(0)$ & 6000 & No & 3.45 & 4.12 & 8 \\
Ke & $1500(\mathrm{NPT})$ & - & $2(0)$ & 7000 & No & 3.72 & 4.56 & 10 \\
Gibbsite & $1450(\mathrm{NVT})$ & 0.931 & $2(0)$ & 8000 & No & 3.5 & 3.05 & 7 \\
Gibbsite & $1000(\mathrm{NPT})$ & - & $2(0)$ & 10000 & No & 3.5 & 3.05 & 7 \\
GsI & $1450(\mathrm{NPT})$ & - & $2(0)$ & 8300 & No & 3.6 & 2.9 & 10 \\
GsK & $1515(\mathrm{NVT})$ & 0.950 & $2(0)$ & 10000 & No & 3.55 & 3.08 & 6 \\
GsK (thick) & $1515(\mathrm{NVT})$ & 0.931 & $1(0)$ & 12500 & No & 3.55 & 3.08 & 8 \\
Ge & $1500(\mathrm{NPT})$ & - & $2(0)$ & 9600 & No & 4.63 & 3.42 & 10
\end{tabular}

Table S6: Summary of NVT simulations performed for $\beta$-AgI and $\mathrm{PbI}_{2}$. The labels are as in Table S5.

\begin{tabular}{ccccccccc} 
Surface Label & $\mathrm{N}$ & $\rho(\mathrm{g} / \mathrm{cc})$ & Trials & Time $(\mathrm{ns})$ & $\mathrm{IN}$ & $\mathrm{x}(\mathrm{nm})$ & $\mathrm{y}(\mathrm{nm})$ & $\mathrm{z}(\mathrm{nm})$ \\
\hline AgI $($ Ag exposed) & $2390(\mathrm{NVT})$ & 0.95 & $3(3)$ & $200(60)$ & Yes & 4.2 & 3.64 & 10 \\
AgI (I exposed) & $2390(\mathrm{NVT})$ & 0.95 & $3(0)$ & 2000 & No & 4.2 & 3.64 & 10 \\
$\mathrm{PbI}_{2}$ (Pb exposed) & $2390(\mathrm{NVT})$ & 0.95 & $3(3)$ & $200(60)$ & Yes & 4.2 & 3.64 & 10 \\
$\mathrm{PbI}_{2}$ (I exposed) & $2390(\mathrm{NVT})$ & 0.95 & $3(0)$ & 2000 & No & 4.2 & 3.64 & 10
\end{tabular}


Table S7: Lattice parameters used for all the surfaces considered, together with the relevant 2D lattice distances. Numbers in bold represent average values. NA indicates measurements not applicable for a particular system.

\begin{tabular}{cccc} 
Surface Label & $\mathrm{a} \times \mathrm{b} \times \mathrm{c}(\AA)$ & $2 \mathrm{D} \Delta$ distance $(\AA)$ & $(\mathrm{L}, \mathrm{W})(\AA)$ \\
\hline Ice $\left(\mathrm{I}_{h}\right)$ & $4.506 \times 4.506 \times 7.346$ & 4.506 & $(9.012,10.119)$ \\
$\alpha$-alumina & $4.754 \times 4.754 \times 12.98$ & 4.754 & $(8.234,9.508)$ \\
Kaolinite/kaolnoSi & $5.154 \times 8.942 \times 7.391$ & $\mathbf{5 . 1 6 8}$ & $(8.942,10.307)$ \\
Gibbiste & $8.684 \times 5.078 \times 9.736$ & $\mathbf{5 . 1 4}$ & $(8.452,10.059)$ \\
Hematite & $5.03 \times 5.03 \times 13.75$ & 5.03 & $(8.712,10.06)$ \\
AsI & $4.506 \times 4.506 \times 12.982$ & 4.506 & $(7.805,9.012)$ \\
AsH & $5.03 \times 5.03 \times 13.75$ & 5.03 & $(8.712,10.06)$ \\
HsI & $4.506 \times 4.506 \times 13.75$ & 4.506 & $(7.805,9.012)$ \\
Hc1 & $4.4 \times 4.4 \times 13.75$ & 4.4 & $(7.621,8.8)$ \\
$\mathrm{Hc} 2$ & $4.32 \times 4.32 \times 13.75$ & 4.315 & $(7.474,8.63)$ \\
$\mathrm{HsA}$ & $4.754 \times 4.754 \times 12.982$ & 4.754 & $(8.234,9.508)$ \\
$\mathrm{KsI}$ & $4.897 \times 9.012 \times 7.391$ & $\mathbf{5 . 0 5 1}$ & $(8.632,10.241)$ \\
$\mathrm{KsG}$ & $5.078 \times 8.684 \times 7.391$ & $\mathbf{5 . 0 4 6}$ & $(8.678,10.06)$ \\
$\mathrm{GsI}$ & $9.012 \times 4.8973 \times 9.736$ & $\mathbf{5 . 1 3}$ & $(8.459,10.233)$ \\
$\mathrm{GsK}$ & $8.942 \times 5.154 \times 9.736$ & $\mathbf{5 . 2 6}$ & $(8.838,10.321)$ \\
$\mathrm{Kc} 1$ & $5.104 \times 8.842 \times 7.391$ & $\mathbf{5 . 1 0 5}$ & $(8.827,10.225)$ \\
$\mathrm{Kc} 2$ & $5.054 \times 8.742 \times 7.391$ & $\mathbf{5 . 0 5 1}$ & $(8.737,10.113)$ \\
$\mathrm{Kc} 3$ & $4.978 \times 8.641 \times 7.391$ & $\mathbf{4 . 9 8 4}$ & $(8.614,9.988)$ \\
$\mathrm{Kc} 4$ & $4.927 \times 8.529 \times 7.391$ & $\mathbf{4 . 9 2 6}$ & $(8.52,9.865)$ \\
$\mathrm{Ke}$ & $5.304 \times 9.173 \times 7.391$ & $\mathbf{5 . 3 0}$ & $(9.169,10.612)$ \\
$\mathrm{Ge}$ & $9.30 \times 5.73 \times 9.736$ & $\mathbf{5 . 6 5}$ & $(9.68,10.92)$ \\
$\beta-\mathrm{AgI}$ & $4.598 \times 4.598 \times 7.514$ & 4.598 & $\mathrm{NA}$ \\
$\mathrm{PbI}{ }_{2}$ & $4.557 \times 4.557 \times 20.94$ & 4.557 & $\mathrm{NA}$ \\
$\mathrm{Boehmite}$ & $2.851 \times 12.12 \times 3.736$ & $\mathrm{NA}$ & $(7.472,8.553)$
\end{tabular}


Table S8: Complete set of lattice mismatch parameters for the basal and prism faces of $\mathrm{I}_{h}$. The $\delta$ values are obtained from Table S2. The $\delta_{2 D}$ values are calculated from the measurements given in Table S7. The values for physical surfaces are also given in the main text. The last column indicates whether or not ice nucleation was observed, and, if so, which ice plane was involved. (Al LJ) indicates that the $\mathrm{Al}$ force field was used.

\begin{tabular}{|c|c|c|c|c|c|}
\hline Surface Label & $\operatorname{Basal}\left(\delta_{2 D} \%\right)$ & $\operatorname{Prism}\left(\delta_{2 D} \%\right)$ & $\operatorname{Basal}(\delta \%)$ & $\operatorname{Prism}(\delta \%)$ & Ice nucleation \\
\hline Ice $\left(\mathrm{I}_{h}\right)$ & 0.0 & 0.0 & 0.0 & 0.0 & - \\
\hline$\alpha$-Alumina & 5.5 & $(8.6,6.6)$ & $(5.5,5.5)$ & $(5.5,2.93)$ & (Y, basal) \\
\hline Kaolinite & 14.6 & $(0.78,1.85)$ & $(14.3,0.8)$ & $(5.16,0.8)$ & (Y, prism) \\
\hline KaolnoSi & 14.6 & $(0.78,1.85)$ & $(14.3,0.8)$ & $(5.16,0.8)$ & (Y, prism) \\
\hline Gibbsite & 14.1 & $(6.2,0.59)$ & $(3.6,12.7)$ & $(3.6,3.69)$ & $\mathrm{N}$ \\
\hline Hematite & 11.6 & $(3.3,0.58)$ & $(11.6,11.6)$ & $(11.6,2.71)$ & $\mathrm{N}$ \\
\hline AsI & 0.0 & $(13.4,10.94)$ & $(0.0,0.0)$ & $(0.0,8.0)$ & (Y, basal) \\
\hline $\mathrm{AsH}$ & 11.6 & $(3.3,0.58)$ & $(11.6,11.6)$ & $(11.6,2.71)$ & $\mathrm{N}$ \\
\hline HsI (Al LJ) & 0.0 & $(13.4,10.94)$ & $(0.0,0.0)$ & $(0.0,8.0)$ & (Y, basal) \\
\hline $\mathrm{Hc} 1$ & 2.35 & $(15.43,13.03)$ & $(2.35,2.35)$ & $(2.35,10.16)$ & (Y, basal) \\
\hline $\mathrm{Hc} 2$ & 4.24 & $(17.07,14.71)$ & $(4.24,4.24)$ & $(4.24,11.89)$ & (Y, basal) \\
\hline $\mathrm{HsA}$ & 5.5 & $(8.6,6.6)$ & $(5.5,5.5)$ & $(5.5,2.93)$ & $\mathrm{N}$ \\
\hline $\mathrm{KsI}$ & 12.09 & $(4.22,1.21)$ & $(8.68,0.0)$ & $(0.0,0.0)$ & $\mathrm{N}$ \\
\hline $\mathrm{KsG}_{\mathrm{s}}$ & 11.98 & $(2.71,0.58)$ & $(12.7,3.6)$ & $(3.69,3.6)$ & (Y, prism) \\
\hline GsI & 13.77 & $(6.14,1.13)$ & $(0.0,8.68)$ & $(0.0,0.0)$ & $\mathrm{N}$ \\
\hline GsK & 16.62 & $(1.93,2.0)$ & $(0.8,14.3)$ & $(0.8,5.16)$ & $\mathrm{N}$ \\
\hline $\mathrm{Kc1}$ & 13.29 & $(2.05,1.05)$ & $(13.27,1.89)$ & $(4.22,1.89)$ & (Y, prism) \\
\hline $\mathrm{Kc} 2$ & 12.09 & $(3.05,0.06)$ & $(12.12,3.0)$ & $(3.2,3.0)$ & $(\mathrm{Y}$, prism $)$ \\
\hline Kc3 & 10.6 & $(4.42,1.29)$ & $(10.47,4.12)$ & $(1.65,4.12)$ & $\mathrm{N}$ \\
\hline $\mathrm{Kc} 4$ & 9.32 & $(5.46,2.51)$ & $(9.34,5.36)$ & $(0.61,5.36)$ & $\mathrm{N}$ \\
\hline $\mathrm{Ke}$ & 17.62 & $(1.74,4.87)$ & $(17.71,1.79)$ & $(8.30,1.79)$ & $\mathrm{N}$ \\
\hline $\mathrm{Ge}$ & 25.4 & $(7.41,7.92)$ & $(3.2,27.16)$ & $(3.2,17.0)$ & $\mathrm{N}$ \\
\hline$\beta-A g I$ & 2.04 & NA & $(2.04,2.04)$ & $(2.04,6.11)$ & (Y, basal) \\
\hline $\mathrm{PbI}_{2}$ & 1.13 & NA & $(1.13,1.13)$ & $(1.13,6.95)$ & (Y, basal) \\
\hline Boehmite & NA & $(17,15.48)$ & $(26.5,17)$ & $(26.5,1.72)$ & $\mathrm{N}$ \\
\hline
\end{tabular}


Table S9: The two $\mathrm{O}_{m}$ - $\mathrm{O}$ and one $\mathrm{O}$ - $\mathrm{O}$ (bold numbers) distances determining the size of the triangular shaped interstitial sites on kaolinite, gibbsite, and related surfaces. There are two sets of numbers for gibbsite type surfaces, because there are two types of interstitial sites. Note that for kaolinite and KsG all three distances are very similar, but this is not true for KsI, gibbsite, GsI, and GsK.

\begin{tabular}{ccc} 
Surface Label & $O_{m} / \mathrm{O}-\mathrm{O}$ Distances & IN \\
\hline Kaolinite & $3.442,3.437, \mathbf{3 . 4 4 7}$ & Yes \\
KaolnoSi & $3.442,3.437, \mathbf{3 . 4 4 7}$ & Yes \\
Gibbsite & $3.452,3.245, \mathbf{3 . 0 4 7}$ & No \\
& $3.142,2.986, \mathbf{3 . 4 5 4}$ & \\
KsI & $3.321,3.474, \mathbf{3 . 3 1 7}$ & No \\
KsG & $3.348,3.379, \mathbf{3 . 3 7 5}$ & Yes \\
GsI & $3.365,3.396, \mathbf{2 . 9 9 3}$ & No \\
& $3.258,2.929, \mathbf{3 . 3 9 6}$ & \\
GsK & $3.304,3.516, \mathbf{3 . 1 0 3}$ & No \\
& $3.235,3.04, \mathbf{3 . 5 1 8}$ & \\
Kc1 & $3.409,3.403, \mathbf{3 . 4 0 7}$ & Yes \\
Kc2 & $3.373,3.370, \mathbf{3 . 3 6 9}$ & Yes \\
Kc3 & $3.325,3.331, \mathbf{3 . 3 2 1}$ & No \\
Kc4 & $3.288,3.288, \mathbf{3 . 2 8 5}$ & No \\
Ke & $3.539,3.536, \mathbf{3 . 5 3 5}$ & No \\
Ge & $3.477,3.846, \mathbf{3 . 3 9 8}$ & No \\
& $3.367,3.332, \mathbf{3 . 8 4 9}$ &
\end{tabular}




\section{References}

(1) Goto, A.; Hondoh, T.; Mae, S. The Electron Density Distribution in Ice $\mathrm{I}_{h}$ Determined by Single-Crystal X-ray Diffractometry. J. Chem. Phys. 1990, 93, 1412-1417.

(2) Malenkov, G. Liquid Water and Ices: Understanding the Structure and Physical Properties. J. Phys. Condens. Matter 2009, 21, 283101.

(3) Maslen, E. N.; Streltsov, V. A.; Streltsova, N. R.; Ishizawa, N.; Satow, Y. Synchrotron X-ray Study of the Electron Density in $\alpha-\mathrm{Al}_{2} \mathrm{O}_{3}$. Acta Crystallogr. B 1993, 49, 973-980.

(4) El Mendili, Y.; Bardeau, J.-F.; Randrianantoandro, N.; Grasset, F.; Greneche, J.-M. Insights into the Mechanism Related to the Phase Transition from $\gamma-\mathrm{Fe}_{2} \mathrm{O}_{3}$ to $\alpha-\mathrm{Fe}_{2} \mathrm{O}_{3}$ Nanoparticles Induced by Thermal Treatment and Laser Irradiation. J. Phys. Chem. C 2012, 116, 23785-23792.

(5) Bish, D. L. Rietveld Refinement of the Kaolinite Structure at 1.5 K. Clays Clay Miner. 1993, 41, 738-744.

(6) Saalfeld, H.; Wedde, M. Refinement of the Crystal Structure of Gibbsite, $\mathrm{Al}(\mathrm{OH})_{3} . Z$. Kristallogr. Cryst. Mater. 1974, 139.

(7) Cava, R.; Reidinger, F.; Wuensch, B. Single-Crystal Neutron-Diffraction Study of AgI Between $23^{\circ}$ and $300^{\circ}$ C. Solid State Commun. 1977, 24, $411-416$.

(8) Zemann, J. Crystal Structures, 2nd ed. Vol. 1 by R. W. G. Wyckoff. Acta Crystallogr. 1965, 18, 139-139.

(9) Bokhimi, X.; Toledo-Antonio, J.; Guzmán-Castillo, M.; Hernández-Beltrán, F. Relationship Between Crystallite Size and Bond Lengths in Boehmite. J. Solid State Chem. 2001, $159,32-40$. 